\title{
Large-scale ${ }^{13} \mathrm{C}$-flux analysis reveals mechanistic principles of metabolic network robustness to null mutations in yeast Lars M Blank, Lars Kuepfer and Uwe Sauer
}

\author{
Address: Institute of Biotechnology, ETH Zürich, 8093 Zürich, Switzerland.
}

Correspondence: Uwe Sauer. E-mail: sauer@biotech.biol.ethz.ch

Published: 17 May 2005

Genome Biology 2005, 6:R49 (doi:10.1 I86/gb-2005-6-6-r49)

The electronic version of this article is the complete one and can be found online at http://genomebiology.com/2005/6/6/R49
Received: I February 2005

Revised: 8 March 2005

Accepted: 6 April 2005

(c) 2005 Blank et al.; licensee BioMed Central Ltd.

This is an Open Access article distributed under the terms of the Creative Commons Attribution License (http://creativecommons.org/licenses/by/2.0), which permits unrestricted use, distribution, and reproduction in any medium, provided the original work is properly cited.

\begin{abstract}
Background: Quantification of intracellular metabolite fluxes by ${ }^{13} \mathrm{C}$-tracer experiments is maturing into a routine higher-throughput analysis. The question now arises as to which mutants should be analyzed. Here we identify key experiments in a systems biology approach with a genome-scale model of Saccharomyces cerevisiae metabolism, thereby reducing the workload for experimental network analyses and functional genomics.
\end{abstract}

Results: Genome-scale ${ }^{13} \mathrm{C}$ flux analysis revealed that about half of the 745 biochemical reactions were active during growth on glucose, but that alternative pathways exist for only $5 \mathrm{I}$ gene-encoded reactions with significant flux. These flexible reactions identified in silico are key targets for experimental flux analysis, and we present the first large-scale metabolic flux data for yeast, covering half of these mutants during growth on glucose. The metabolic lesions were often counteracted by flux rerouting, but knockout of cofactor-dependent reactions, as in the $a \mathrm{dh} l$, ald 6 , $\operatorname{cox} 5 \mathrm{~A}$, fum I, mdhI, pdal, and zwfl mutations, caused flux responses in more distant parts of the network. By integrating computational analyses, flux data, and physiological phenotypes of all mutants in active reactions, we quantified the relative importance of 'genetic buffering' through alternative pathways and network redundancy through duplicate genes for genetic robustness of the network.

Conclusions: The apparent dispensability of knockout mutants with metabolic function is explained by gene inactivity under a particular condition in about half of the cases. For the remaining 207 viable mutants of active reactions, network redundancy through duplicate genes was the major (75\%) and alternative pathways the minor (25\%) molecular mechanism of genetic network robustness in S. cerevisiae.

\section{Background}

The availability of annotated genomes and accumulated biochemical evidence for individual enzymes triggered the reconstruction of stoichiometric reaction models for network-based pathway analysis $[1,2]$. For many microbes, such network models are available at the genome scale, providing a largely comprehensive metabolic skeleton by interconnecting all known reactions in a given organism $[3,4]$. Thus, network properties such as optimal performance, flexibility to cope with ever-changing environmental conditions, and 
enzyme dispensability (also referred to as robustness or genetic robustness $[5,6]$ ) become mathematically tractable. These computational advances are matched with postgenomic advances in experimental methods that assess the cell's molecular make-up at the level of mRNA, protein, or metabolite concentrations. As the functional complement to these compositional data, quantification of intracellular in vivo reaction rates or molecular fluxes has been a focal point of method development in the realm of metabolism [7-9]. Recent progress in increasing the throughput of stable-isotope-based flux analyses $[8,10,11]$ has allowed the quantification of flux responses to more than just a few intuitively chosen genetic or environmental perturbations [12-14]. Now that flux quantification in hundreds of null mutants under a particular condition is feasible in principle, the question arises of which mutants should be analyzed.

As perhaps the most widely used model eukaryote, the yeast Saccharomyces cerevisiae features a metabolic network of about 1,200 reactions that represent about 750 biochemically distinct reactions [3,15]. Is it necessary to quantify flux responses to null mutations in all reactions for a comprehensive view of the metabolic capabilities under a given condition? To address this question, we used a recently modified version (iLL672; L Kuepfer, U Sauer and LM Blank, unpublished work) of the original iFF708 genome-scale model published by Förster et al. [3]. On the basis of this model, we estimated the genome-scale flux distribution in wild-type $S$. cerevisiae from ${ }^{13 \mathrm{C}}$-tracer experiments, to identify the 339 biochemical reactions that were active during growth on glucose. Yeast metabolism has the potential flexibility to use alternative pathways for 105 of these active reactions. For a major fraction of the potentially flexible reactions that catalyze significant flux, we then constructed prototrophic knockout mutants to elucidate whether or not the alternative pathway was used upon experimental knockout; that is, whether it contributes to the genetic robustness of the network $[5,6]$. For the purpose of this work, robustness is defined as the ability to proliferate on glucose as the sole carbon source upon knockout of a single gene with metabolic function.

\section{Results}

Identification of flexible reactions in yeast metabolism To identify all potentially flexible reactions in yeast glucose metabolism that were active under a given condition, we used the recently reconciled metabolic network model iLL672 with 1,038 reactions (encoded by 672 genes) that represent 745 biochemically distinct reactions (L Kuepfer, U Sauer and LM Blank, unpublished work), which was based on the genomescale $S$. cerevisiae model iFF708 [3]. The main modifications to the original model include elimination of dead-end reactions and a new formulation of cell growth. It should be noted that none of the results below critically depended on the network model, but the reconciliation of iLL672 enabled a more

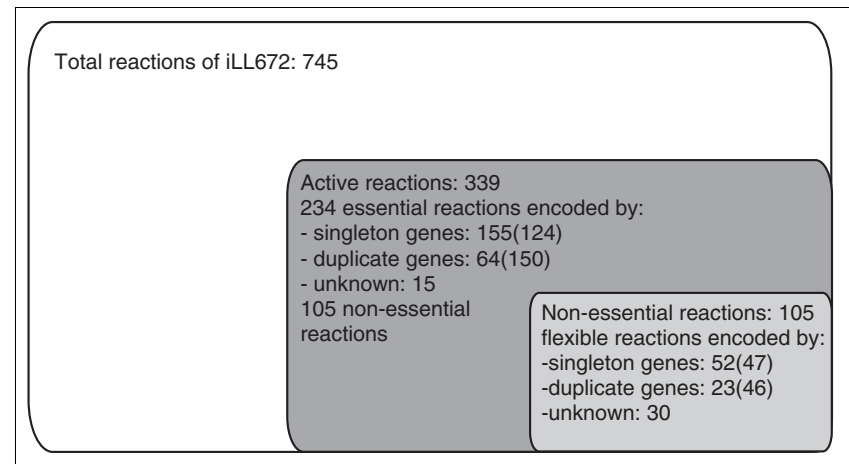

Figure

Genome-wide proportion of active, essential and flexible metabolic reactions during growth of S. cerevisiae (iLL672) on glucose. Flexible reactions are defined as having a non-zero flux but are not essential for growth. The number of genes that encode biochemical reactions is given in parentheses.

accurate discrimination between lethal and viable reactions than iFF708, as was validated by large-scale growth experiments (L Kuepfer, U Sauer and LM Blank, unpublished work).

First, we identified all reactions active in wild-type glucose metabolism by genome-scale flux analysis. For this purpose, we determined the wild-type flux distribution in central metabolism from a stable isotope batch experiment with $20 \%$ [U-13C] and $80 \%$ unlabeled glucose. This flux solution was then mapped to the genome scale by using minimization of the Euclidean norm of fluxes as the objective function. In total, 339 of the 745 biochemical reactions were active during growth on glucose alone (Figure 1 and Additional data file 1), which agrees qualitatively with the estimate of Papp et al. [16]. Most active reactions (234) were essential: 155 are encoded by singleton genes, 64 by two or more duplicate genes and 15 by yet unknown genes (Figure 1; Additional data file 1). In the entire network, only the remaining 105 reactions (30 encoded by yet unknown genes) were active and potentially flexible in the sense that they may be bypassed via alternative pathways (Figure 1). As fluxes in the peripheral reactions were typically below $0.1 \%$ of the glucose uptake rate (see Additional data file 1), we focused on the 51 geneencoded flexible reactions that catalyzed a flux of at least $0.1 \%$. These 51 reactions were encoded by 75 genes (43 duplicates, 23 singletons and 9 multiprotein complexes).

\section{Physiological fitness of mutants deleted in flexible reactions}

In 38 of these genes, which encoded 28 of the 51 potentially flexible and highly active reactions, we constructed prototrophic deletion mutants by homologous recombination [17] in the physiological model strain CEN.PK [18] (Figure 2). The prototrophic background was chosen to minimize potential problems of amino-acid supplementation for quantitative analysis [19]. These 38 experimental knockouts were in the 
Table I

Fitness of mutants with deletions in flexible central metabolic reactions

\begin{tabular}{|c|c|c|c|c|c|c|c|}
\hline \multirow[b]{2}{*}{ Mutants } & \multicolumn{2}{|c|}{ Physiological fitness* } & \multirow{2}{*}{$\frac{\text { Competitive fitness }}{\text { YPD }}$} & \multirow[b]{2}{*}{ Mutants } & \multicolumn{2}{|c|}{ Physiological fitness } & \multirow{2}{*}{$\frac{\text { Competitive fitness }}{\text { YPD }}$} \\
\hline & MM & YPD & & & MM & YPD & \\
\hline Reference strain & 1 & 1 & 1 & & & & \\
\hline adh I/YOL086C & 0.47 & 0.57 & 0.79 & $m d h 2 / Y O L I 26 C$ & 0.89 & 0.98 & 1.01 \\
\hline adh3/YMR083W & 0.92 & 0.87 & 0.98 & mdh3/YDL078C & 1.00 & 0.96 & 1.01 \\
\hline ald5/YER073W & 1.02 & 0.94 & 1 & $\mathrm{mls} I / Y N L I I 7 \mathrm{~W}$ & 1 & 0.98 & 1 \\
\hline ald6/YPL06IW & 0.34 & 0.87 & 0.9 & oacl/YKLI20W & 0.71 & 0.94 & 1.01 \\
\hline $\operatorname{cox} 5 \mathrm{~A} / \mathrm{YNL} 052 \mathrm{~W}$ & 0.63 & 0.91 & 1 & pckI/YKR097W & 1 & 0.96 & 1 \\
\hline ctp//YBR29IC & 0.91 & 1 & 0.97 & pdal/YERI78W & 0.41 & 0.98 & 1 \\
\hline dal7/YIR03IC & 0.94 & 0.85 & 1 & pgm I/YKLI27W & 0.82 & 0.94 & 1 \\
\hline fum I/YPL262W & 0.52 & 0.62 & 0.93 & pgm2/YMRI05C & 0.90 & 1 & 1 \\
\hline gnd I/YHRI83W & 0 & 0.87 & 1.01 & rpel/YJLI2IC & 0.33 & 0.94 & 0.88 \\
\hline gnd2/YGR256W & 0.83 & 0.98 & 1 & sdhl/YKLI48C & 0.72 & 0.94 & 1 \\
\hline gcv2/YMRI89W & 0.92 & 0.94 & 1 & ser33/YIL074C & 0.92 & 0.94 & 1.01 \\
\hline glyl/YEL046C & 0.79 & 0.74 & 0.87 & sfcl/YJR095W & 0.84 & 0.96 & 1.01 \\
\hline gpdI/YDL022W & 1 & 0.98 & 0.84 & soll/YNR034W & 0.91 & 1 & 1.02 \\
\hline icll/YER065C & 1 & 1 & 1 & sol2/YCRXI3W & 0.99 & 0.98 & 1 \\
\hline idp I/YDL066W & 0.92 & 0.94 & 1.03 & sol3/ YHRI63W & 0.71 & 0.94 & 1 \\
\hline idp2/YLRI74WW & 0.86 & 0.96 & 0.95 & sol4/ YGR248W & 0.95 & 0.91 & 1.01 \\
\hline Iscl/YORI42W & 1.05 & 0.93 & 1 & tall/ YLR354C & 0.89 & 0.94 & 1 \\
\hline mael/YKL029C & 1.01 & 0.96 & 1 & YGR043C & 0.92 & 0.87 & 1.02 \\
\hline mdh I/YKL085W & 0.72 & 0.91 & 1 & zwfI/YNL24IC & 0.38 & 0.96 & ND \\
\hline
\end{tabular}

*Physiological fitness is defined as the maximal specific growth rate of a mutant normalized to the reference strain CEN.PK II3-7D ho::kanMX4. The average from triplicate experiments is shown. The standard deviation was generally below 0.05. FFrom Steinmetz et al. [20]. ND, not detected.

pentose phosphate (PP) pathway, tricarboxylic acid (TCA) cycle, glyoxylate cycle, polysaccharide synthesis, mitochondrial transporters, and by-product formation (Figure 2, Table 1). Genetically, the knockouts encompass 14 singleton and 24 duplicate genes, including six gene families of which all members were deleted.

With the exception of gnd1, all 38 mutants grew with glucose as the sole carbon source. The lethal phenotype of the gnd 1 mutant is consistent with a previous report [20] and is similar to the gndA mutant in Bacillus subtilis [21]. As in B. subtilis, we could select gnd 1 suppressor mutants on glucose (data not shown). To assess the quantitative contribution of each gene to the organism's fitness, we determined maximum specific growth rates in minimal and complex medium using a wellaerated microtiter plate system [22]. Mutant fitness was then expressed as the normalized growth rate, relative to the growth rate of the reference strain (Table 1). In contrast to the previously reported competitive fitness $[20,23,24]$, the fitness determined here is a quantitative physiological value.

In complex YPD medium, physiological fitness in the 38 viable haploid mutants was generally in qualitative agreement with the competitive fitness [20]. Quantitatively, however, our data seem to allow a better discrimination (Table 1), and significant differences between physiological and competitive fitness were seen in the adh1, fum1, and gpd1 mutants. Only threemutants - adh1, fum1, and gly1 - exhibited a fitness defect of $20 \%$ or greater (Table 2). gly1 lacks threonine aldolase, which catalyzes cleavage of threonine to glycine [25], hence its phenotype remains unexplained because glycine was present in the YPD medium.

Figure 2 (see following page)

Central carbon metabolism of $\mathrm{S}$. cerevisiae during aerobic growth on glucose. Gene names in boxes are given for reactions that were identified as flexible by flux balance analysis. Dark gray boxes indicate mutants, for which the carbon flux distribution was determined by ${ }^{13} \mathrm{C}$-tracer experiments. Dots indicate that the gene is part of a protein complex. Arrowheads indicate reaction reversibility. Extracellular substrates and products are capitalized. $\mathrm{CI}$, onecarbon unit from $\mathrm{C}_{1}$ metabolism. 


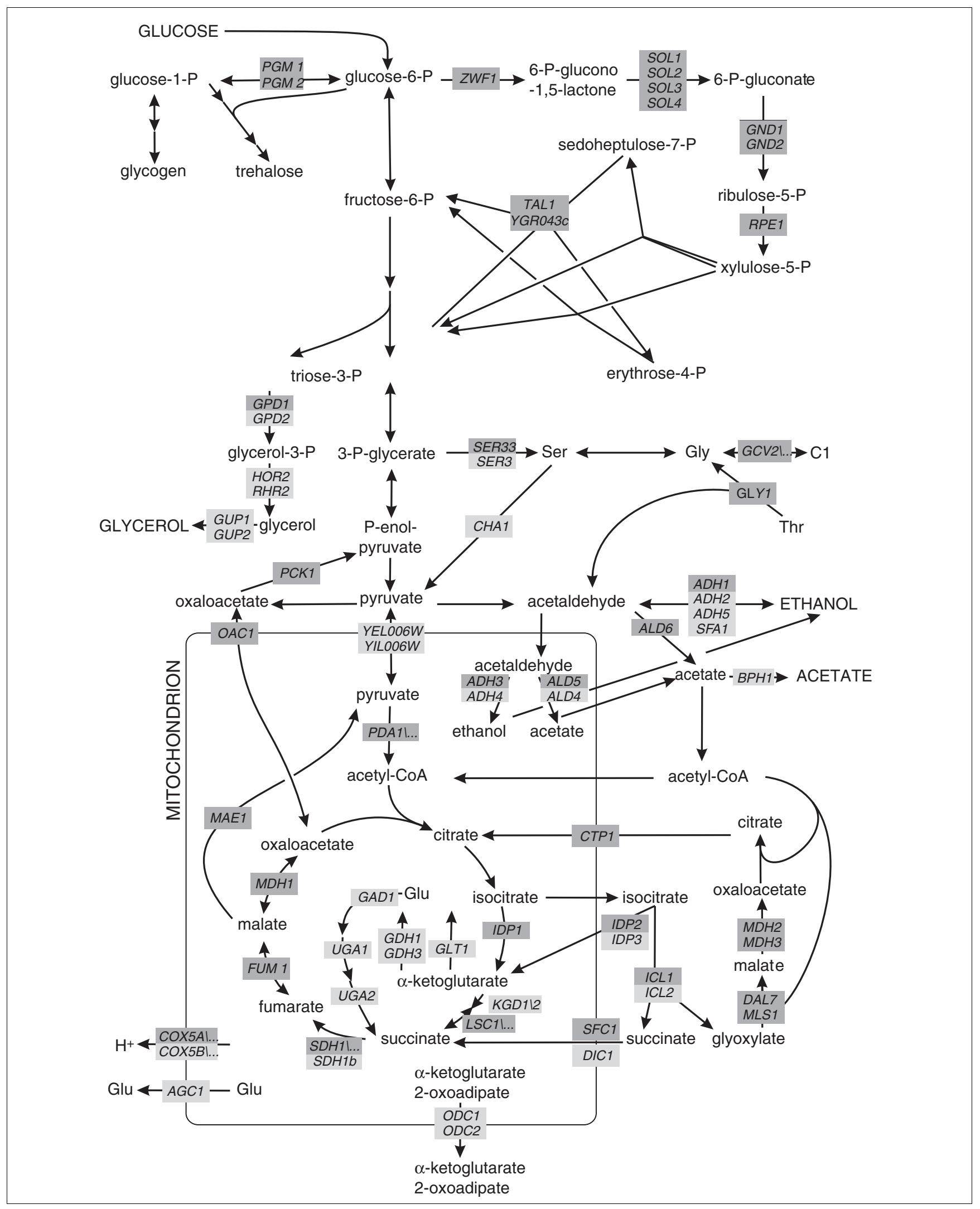

Figure 2 (see legend on previous page) 
Table 2

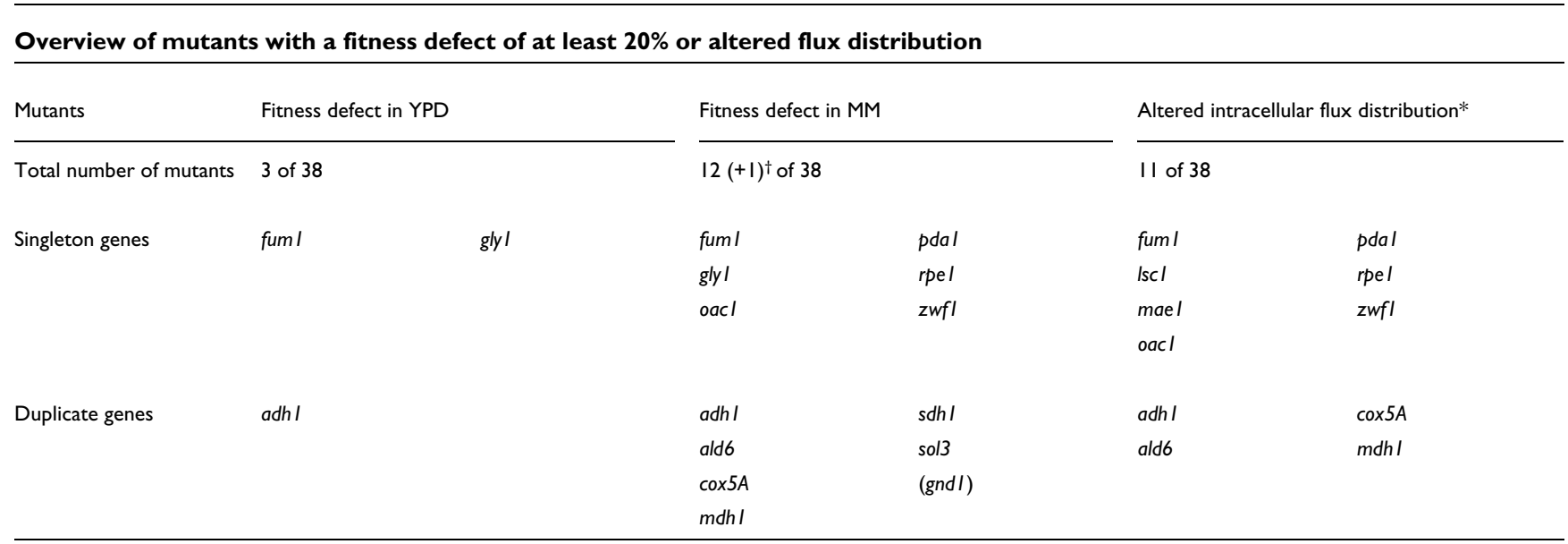

*See Figures 5 and 6. tLethal mutations are given in parentheses.

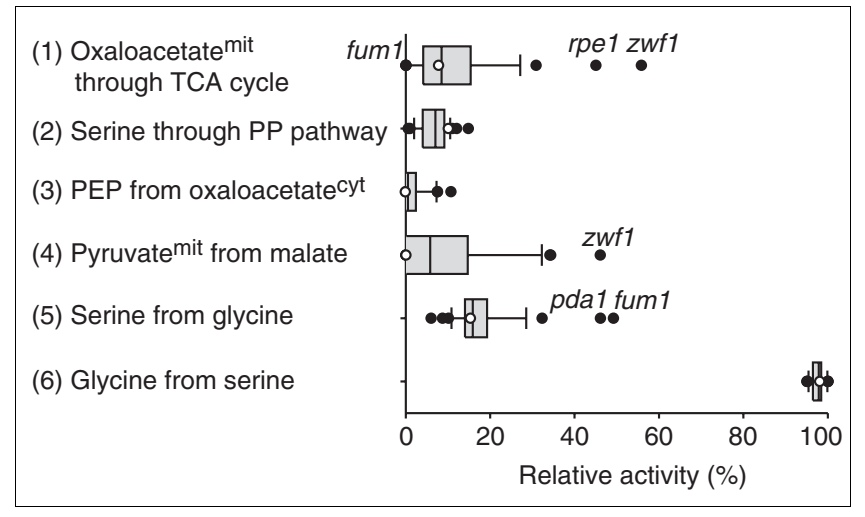

Figure 3

The distribution of six independently determined metabolic flux ratios in 37 deletion mutants during growth on glucose. In each case, the median of the distribution is indicated by a vertical line, the 25 th percentile by the grey box and the 90 th percentile by the horizontal line. Data points outside the 90 th percentile are indicated by dots. The reference strain is indicated by the open circle.

In general, growth on the single substrate reduced the metabolic flexibility, as a much greater proportion of mutants exhibited significant fitness defects (Table 2). Major fitness defects were prominent in mutants of the PP pathway (gnd1, rpe1, sol3, and zwf1), which indicates an increased demand of $\mathrm{NADPH}$ for biosynthesis. Fitness of the fum1 mutant was clearly lower than that of other TCA-cycle mutants, for which duplicate genes exist. The strong phenotype of the fum 1 mutant was somewhat unexpected because the flux through the TCA cycle is generally low or absent in glucose batch cultures of $S$. cerevisiae $[13,14,26,27]$.

\section{Intracellular carbon flux redistribution in response to gene deletions}

While physiological data quantify the fitness defect, they cannot differentiate between intracellular mechanisms that bring about robustness to the deletion. To identify how carbon flux was redistributed around a metabolic lesion, we used metabolic flux analysis based on ${ }^{13 C}$-glucose experiments [8,9]. In contrast to in vitro enzyme activities and expression data, 13C-flux analysis provides direct evidence for such in vivo flux rerouting or its absence. The flux protocol consists of two distinct steps: first, analytical identification of seven independent metabolic flux ratios with probabilistic equations from the ${ }_{13} \mathrm{C}$ distribution in proteinogenic amino acids [12,28,29]; and second, estimation of absolute fluxes (in vivo reaction rates) from physiological data and the flux ratios as constraints [10,30]. The relative distribution of intracellular fluxes was rather invariant in the 37 mutants, with the fraction of mitochondrial oxaloacetate derived through the TCA cycle flux and the fraction of mitochondrial pyruvate originating from malate as prominent exceptions (Figure 3).

Figure 4 (see following page)

Absolute metabolic fluxes in the 37 flexible mutants as a function of glucose uptake rate or selected intracellular fluxes. (a-f) Glucose uptake rate; (g,h) selected intracellular fluxes. The linear regression of the distribution and the $99 \%$ prediction interval are indicated by the solid and dashed lines, respectively. Mutants with significant changes in the carbon-flux distribution are indicated. The reference strain is indicated by an open circle. Extreme flux patterns were verified in $30-\mathrm{ml}$ shake flask cultures (data not shown). 
(a)



(c)

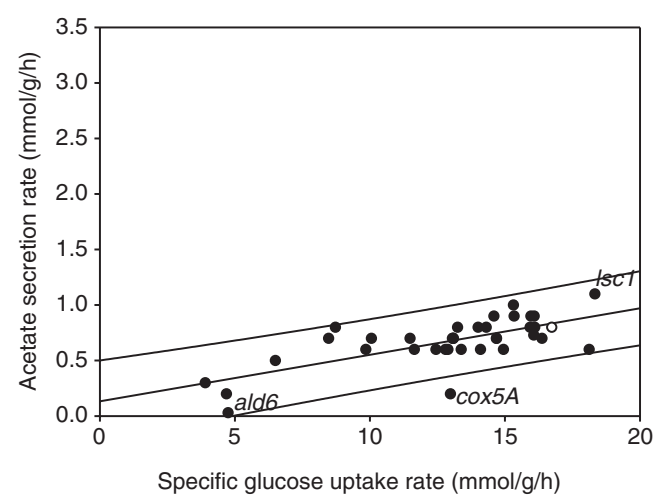

(e)

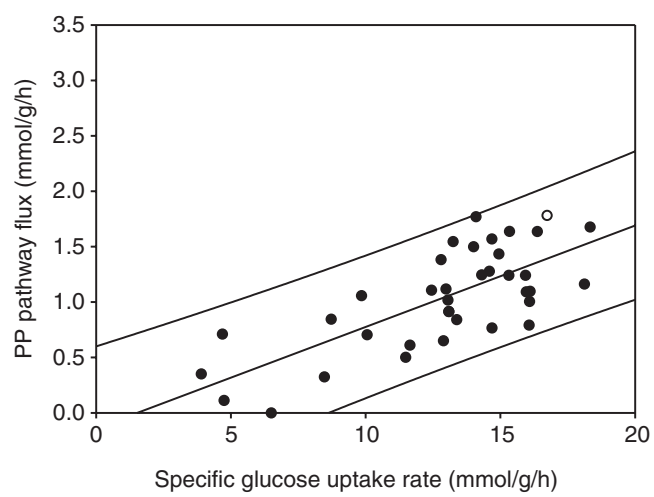

(g)

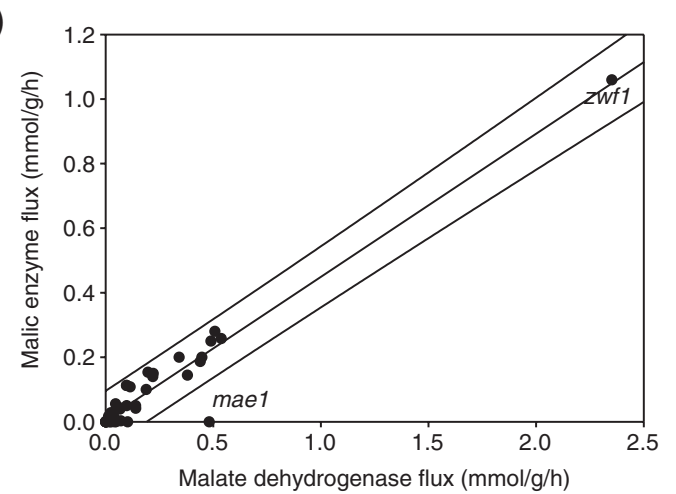

(b)

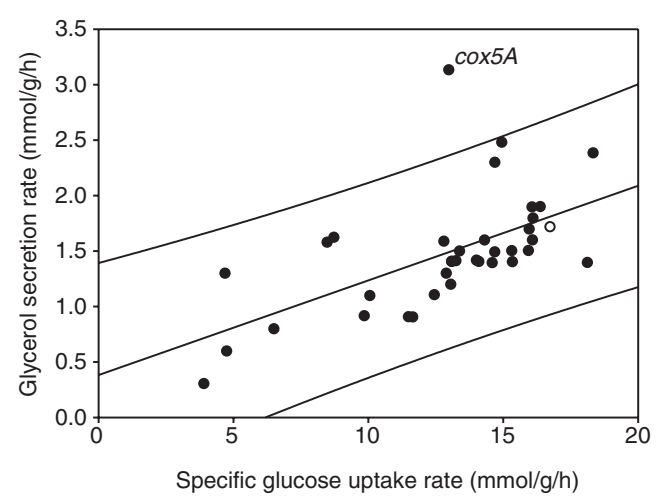

(d)

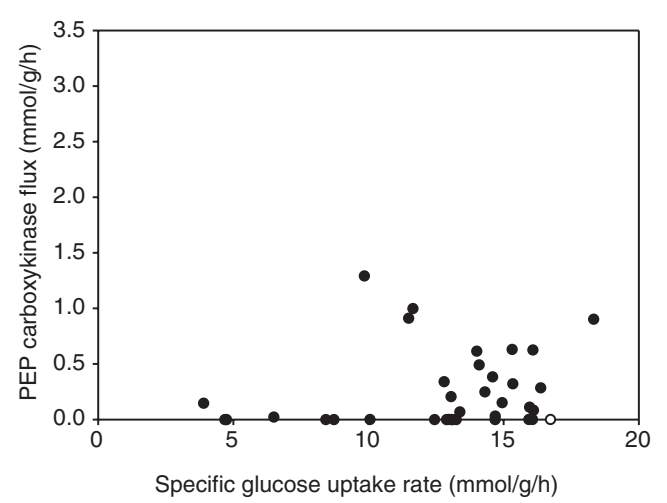

(f)

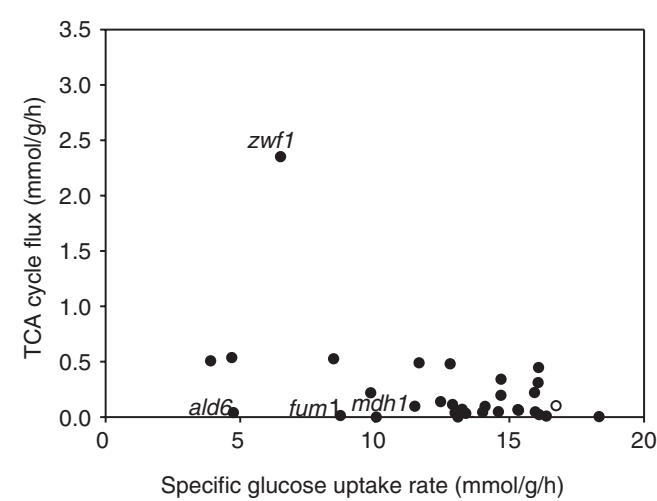

(h)

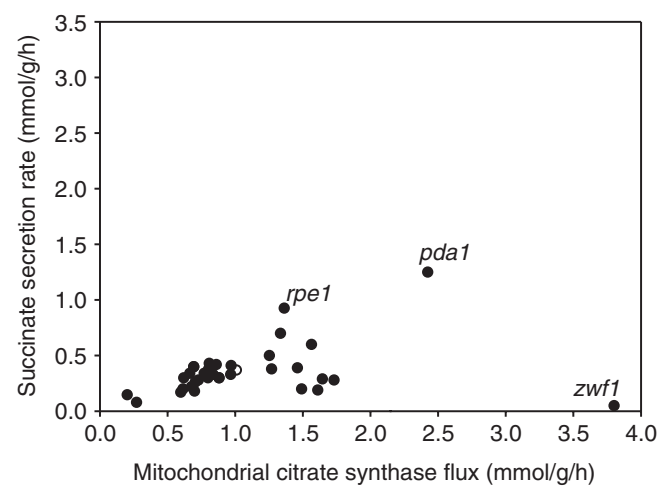

Figure 4 (see legend on previous page) 
From the experimentally determined uptake/production rates and the flux ratios as constraints (Additional data file 3), absolute intracellular fluxes were calculated using a compartmentalized stoichiometric model that consists of 35 reactions and 30 metabolites (Additional data file 2). This flux model comprised mostly the reactions of central carbon metabolism that were most relevant to the genetic changes introduced. It should be noted that the deleted reactions, with the exception of pyruvate dehydrogenase (PDA1), were not omitted from the network model; thus the calculated absence of flux through a given reaction was independently verified from the 13C-labeling data. In contrast to the relative distribution of intracellular fluxes, absolute reaction rates varied significantly in the mutants. With the exception of the flux through the TCA cycle (Figure 4f) and the gluconeogenic PEP carboxykinase (Figure 4d), all other fluxes generally increased with increasing glucose uptake rates (Figure 4). Eleven of the 37 mutants, however, exhibited specific flux responses that deviated from this general trend (Table 2, Figure 4).

\section{Specific flux responses in singleton and duplicate gene knockouts}

Specific flux responses were more prominent among the singleton mutants (Table 2, Figure 4). Although the TCA cycle flux through the $\mathrm{NAD}^{+}$-dependent fumarase reaction from fumarate to malate was already very low in the reference strain (Figures 3, 4f), the fum1 mutant exhibited a pronounced phenotype with altered redox metabolism and significant glycerol production (Figure 5). Inactivation of the mitochondrial pyruvate dehydrogenase complex in the pda1 mutant was bypassed by the import of cytosolic acetyl-CoA into the mitochondria. Inactivation of the oxidative PP pathway branch in the $z w f 1$ mutant was compensated by a reversed flux in the non-oxidative $\mathrm{PP}$ pathway to provide the biomass precursors pentose 5-phosphate and erythrose 4phosphate (Figure 5). Because the primary role of the PP pathway on glucose is generation of NADPH, NADP+dependent mitochondrial malic enzyme flux was significantly increased in the zwf1 mutant. This NADPH compensation by malic enzyme was also suggested recently from co-feeding experiments [31].
In contrast to singletons, deletion of flexible duplicate genes could be compensated by either alternative pathways or isoenzymes. In most cases, however, the isoenzymes were used because no flux alteration was detected, with the a $d h 1$, ald6, $\operatorname{cox} 5 A$, and $m d h 1$ mutants as exceptions (Table 2). Deletion of the major acetate-producing acetaldehyde dehydrogenase, the cytoplasmic ALD6 [32], significantly reduced acetate formation. The primary effect of the deletion was the strongly reduced glucose-uptake rate (Figure 4). Although a major source of NADPH was inactivated in this mutant [33], the PP pathway flux was not increased, but was even lower than in the reference strain (Figure 6). This indicates that the strongly decreased fitness of the ald6 mutant (Table 1) could result from NADPH starvation - that is, a suboptimal rate of $\mathrm{NADP}^{+}$reduction. Consistent with this, we estimated that the $\mathrm{NADPH}$ requirement exceeded the combined NADPH formation from the oxidative PP pathway and malic enzyme by $70 \%$, indicating that an as-yet-unidentified reaction(s) substitutes for the remaining NADPH production. Candidates are the mitochondrial acetaldehyde dehydrogenase Ald $4 \mathrm{p}$ [34], which can use either $\mathrm{NAD}^{+}$or $\mathrm{NADP}^{+}$as redox cofactors or the mitochondrial NADH kinase Pos5p [35]. Deletion of the cytochrome $c$ oxidase subunit $\mathrm{Va} C_{5} X_{5} A$ in the mitochondrial respiratory chain increased glycerol production, which serves as means to reoxidize $\mathrm{NADH}$ (Figures $4 \mathrm{~b}, 6$ ). Because this mutant lacks functional mitochondria [36], glycerol production was driven by the limited NADH reoxidation through residual $\mathrm{NADH}$ oxidase activity in the electron-transport chain. Thus, robustness was brought about by using an alternative NADH sink. Considering that the flux through the mitochondrial malate dehydrogenase Mdh1 was already very low in the reference strain, the fitness defect of the mdh1 was surprising. Akin to the fum1 and ald6 mutants, the significantly reduced fitness of mdh1 may be explained by the imbalance between the TCA cycle and glucose catabolism (Figure 4f). Generally, the TCA cycle flux increases with decreasing glucose uptake rates [29], but remains non-proportionally low (absent) in the fum1, ald6, and mdh1 mutants (Figure 4f). The cytosolic and peroxisomal duplicate genes $\mathrm{MDH} 2$ and $\mathrm{MDH}_{3}$, respectively, did not compensate for the mitochondrial lesion, which is consistent with the observed lethal phenotype of the mdh1 mutant when grown on acetate [37].

\footnotetext{
Figure 5 (see following page)

Relative distributions of absolute carbon fluxes in the S. cerevisiae reference strain (Ref) and the singleton gene mutants fum I, pdal and zwf. All fluxes are normalized to the specific glucose uptake rate, which is shown in the top inset, and are given in the same order in each box. Reactions encoded by deleted genes are shown on a black background, but were not removed from the flux model (except for PDAI). The NADPH balance that is based on the quantified fluxes and the known cofactor specificities is given as a synthetic transhydrogenase flux. In general, the $95 \%$ confidence intervals were between 5 and $10 \%$ for the major fluxes. Larger confidence intervals were estimated for reactions with low flux such as malic enzyme and PEP carboxykinase. Flux distributions were verified in 30-ml shake flask cultures (data not shown). $\mathrm{Cl}$, one-carbon unit from $\mathrm{C}_{\mid}$metabolism; P5P, pentose 5-phosphates.
} 


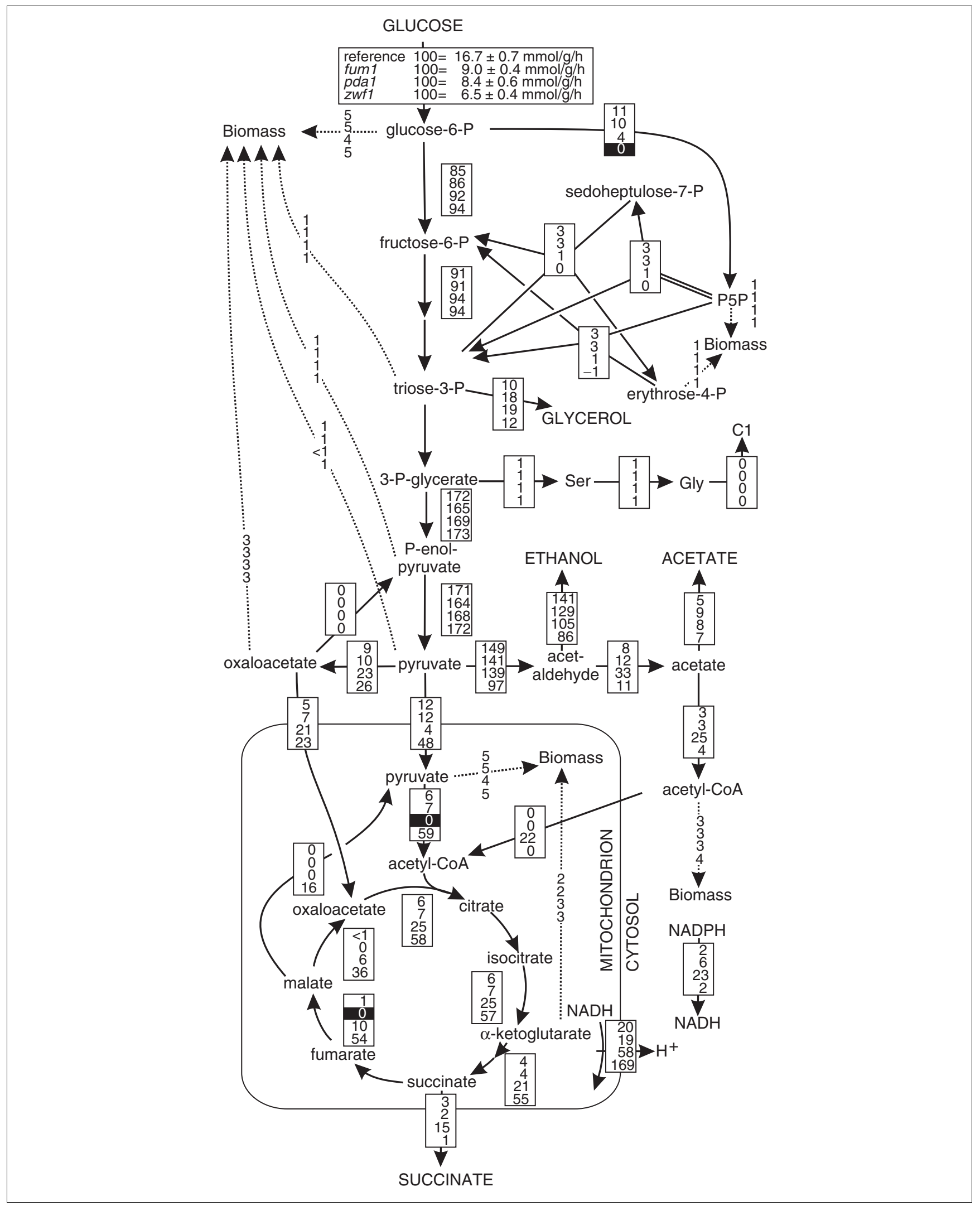

Figure 5 (see legend on previous page) 
Table 3

Overview of mechanisms of metabolic flexibility that confer robustness to central metabolic deletions

\begin{tabular}{|c|c|c|c|}
\hline Duplicate gene* & $\begin{array}{l}\text { Duplicate gene and alternative } \\
\text { pathway }{ }^{\dagger}\end{array}$ & Alternative pathway $\ddagger$ & None \\
\hline $\begin{array}{l}\text { ADH3, ALD5, DAL7, GPD I,ICLI, } \\
I D P I, I D P 2, M D H 2, M D H 3, M L S I,\end{array}$ & ADHI, ALD6, COX5A,MDHI & $\begin{array}{l}\text { FUMI, GLYI, LSCI, MAEI, MDHI, } \\
\text { OACI, PCKI, PDAI, RPEI, ZWFI }\end{array}$ & CTPI, GCV2, GNDI§, GND2, SFCI \\
\hline
\end{tabular}

PGMI, PGM2, SDHI, SER33,SOLI,

SOL2, SOL3, SOL4, TALI, YGR043C

*Wild-type-like flux distribution. †Altered flux distribution, but some residual flux through the reaction was observed. $\ddagger$ Altered flux distribution, but no residual flux through the reaction was observed. § Lethal, probably because of a non-stoichiometric effect.

\section{Genetic network robustness}

The above flux results reveal that knockouts of flexible reactions are bypassed through alternative pathways in about one third of the cases and through isoenzymes in the other two thirds. Does this reflect the relative contribution of alternative pathways and duplicate genes to genetic network robustness? [5] To address this question quantitatively for glucose metabolism, we grew the 196 duplicate (encoding 87 reactions) and 171 singleton (encoding 207 reactions) knockout mutants of all 294 gene-encoded active reactions on glucose plates.

In the 47 viable singleton knockouts, flux rerouting through an alternative pathway ensures survival, which was directly verified by flux data in 10 cases (Figure 4, Table 3 and Additional data file 3). Of the 196 experimental duplicate mutants, 180 grew on sole glucose, while 16 of the mutations were lethal. As these 16 duplicates obviously did not contribute to genetic robustness, their entire families (36 genes) were subtracted from the 150 duplicate-encoded essential reactions (Figure 1). For the remaining 114 duplicate genes we have strong evidence for network redundancy as the underlying mechanism of robustness, because they encode essential reactions (as determined in silico) and each of the experimental knockouts was viable (Figure 7). For the 46 duplicate genes that encode flexible reactions (Figure 1), both compensation by duplicates and/or alternative pathways might ensure proliferation. Where available, these mutants were classified according to their flux distribution; that is, of the 24 experimental duplicate mutants analyzed, four used alternative pathways and 20 an isoenzyme (Figure 4, Table 3 and Additional data file 3 ). In total we analyzed all 367 experimental mutants that encode the 294 active reactions of glucose metabolism, 140 of which were lethal and 227 viable. For the vast majority of the viable mutants, we identified the molecular mechanism that brought robustness to the knockout about: about $25 \%$ were alternative pathways and $75 \%$ duplicate genes (Figure 7).

\section{Discussion}

Using an integrated computational and experimental approach, we show here that metabolic flexibility to knockout mutations is restricted to a relatively small set of biochemical reactions. About a third of all active reactions under the particular condition investigated may be bypassed by alternative pathways, of which about 30\% support only negligible fluxes. The occurrence of flexible reactions might be even lower in prokaryotes, because several alternative pathways involved inter-compartmental transport. In general, the number of flexible reactions will differ substantially between species, with free-living yeast and fungi at the upper end of the scale, and intracellular pathogens with highly reduced genomes at the lower end.

\footnotetext{
Figure 6 (see following page)

Relative distributions of absolute carbon fluxes in the $S$. cerevisiae reference strain and the duplicate gene mutants ald6, cox $5 \mathrm{~A}$ and $m d h I$. All fluxes are normalized to the specific glucose uptake rate, which is shown in the top inset, and are given in the same order in each box. Reactions encoded by deleted genes are shown on a black background, but were not removed from the flux model. The NADPH balance that is based on the fluxes and the known cofactor specificities is given as a synthetic transhydrogenase. In general, the $95 \%$ confidence intervals were between 5 and I0\% for the major fluxes. Larger confidence intervals were estimated for reactions with low flux such as malic enzyme and PEP carboxykinase. Flux distributions were verified in 30 $\mathrm{ml}$ shake flask cultures (data not shown). $\mathrm{Cl}$, one-carbon unit from $\mathrm{C}_{1}$ metabolism; P5P, pentose 5-phosphates.
} 




Figure 6 (see legend on previous page) 


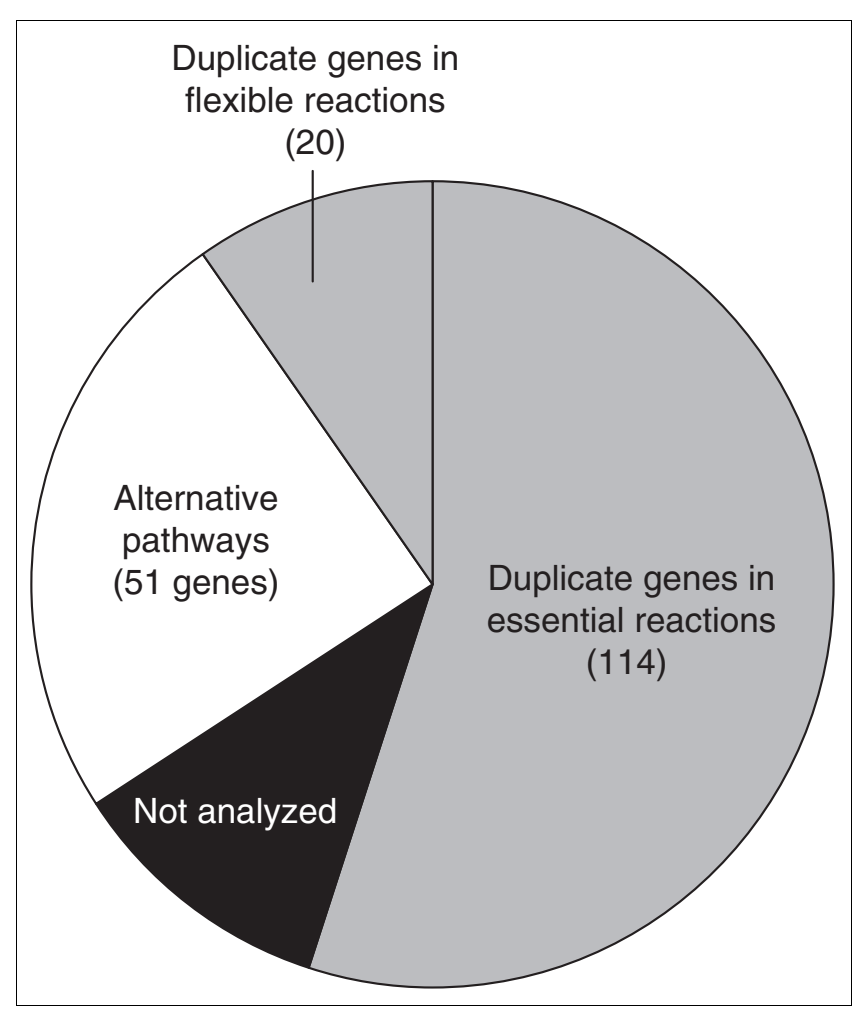

Figure 7

The mechanistic basis of gene dispensability in all active reactions during glucose metabolism of $S$. cerevisiae. The mechanism was mostly identified from the phenotype on glucose plates. For 10 of the alternative pathways and for 20 duplicates encoding flexible reactions, the results were confirmed by ${ }^{13} \mathrm{C}$-flux analysis. For 22 duplicate genes the data are not sufficient to distinguish between both mechanisms and they are labeled as not analyzed.

Using flux balance (FBA) [1,2], elementary flux mode [38,39], or similar analyses [40], all in silico flexible reactions can be precisely identified. Hence, experimental analysis of intracellular flux responses to metabolic gene deletions can be limited to these potentially flexible mutants, rather than having to analyze the entire mutant collection. Using the systems biological approach described here, the true in vivo capability of metabolic network operation can be mapped with a reasonable workload. As the knowledge base on intracellular flux responses increases, a handful of flux experiments in computationally identified mutants will probably suffice to identify the in vivo network capability under a given condition. At the next level, such flux analyses will also include mutants affected in regulatory genes that modulate the network composition. Although not covered in the stoichiometric models employed for flux-balance analysis, several recently discussed computational approaches $[39,41,42]$ may aid in identifying the most relevant regulatory mutants for in vivo flux quantification.

Consistent with the notion that metabolic networks undergo minimal flux redistributions with respect to the metabolic state of the parent [40], deletion of flexible singleton genes was mostly counteracted by local flux rerouting, for example, the lsc1,mae1, and oac1 mutants (see Additional data file 3). Deletions in redox cofactor-dependent singleton or duplicate reactions such as those mediated by adh1, ald6, cox $5 A$, pda1, and $z w f 1$, however, affected flux alterations in more distant reactions. While the relative flux distribution (in \% values) was perturbed only very little in these mutants, the absolute magnitude of fluxes (in vivo reaction velocities) varied dramatically. In particular, knockout of fum1, whose encoded protein catalyzes only a rather small flux, led to an unexpectedly strong phenotype with about a 50\% reduction in glucoseuptake rate. Although unexpected, this finding was qualitatively consistent with results from a recent genetic footprinting study [43], which also showed a significant fitness defect in this mutant. It was speculated that intramitochondrial shortage of amino acids such as aspartate and glutamate causes a lack of respiratory chain components, which leads to a petite-like phenotype [44]. Another key mutant was pda1, whose knockout caused a substantial import of acetyl-CoA into the mitochondria; the mechanism for this remains elusive because the carnitine auxotrophic CEN.PK strain does not use the carnitine shuttle [45]. As a consequence, a twofold overproduction of NADPH was estimated, which suggests that the $\mathrm{NAD}^{+}$-dependent acetaldehyde dehydrogenases instead of the $\mathrm{NADP}^{+}$-dependent $A L D 6$ were active to balance $\mathrm{NADPH}$ formation/consumption. Consistent with this, the flux through the NADPH-producing PP pathway was significantly lower in this mutant. The strongly altered redox metabolism in pda1 is further evidenced by the substantial secretion of glycerol and succinate (Figure 5).

The metabolic flexibility to cope with metabolic lesions is generally known as genetic robustness [5], a concept that is used to explain the seemingly surprising number of phenotypically silent deletion mutations: only about 1,100 knockouts of the 5,700 genes are lethal in haploid $S$. cerevisiae [23,46]. The causes and evolution of gene dispensability have been investigated in several theoretical analyses of pre-existing data, but the issue remains controversial [5,6,16,47-49]. For metabolic networks, our flux data differentiate between the relative contributions of three mechanisms to the apparent genetic robustness: inactive, and thus dispensable, genes; 'genetic buffering' through alternative reactions; and functional complementation from duplicate genes ('redundancy').

\section{Conclusions}

In qualitative agreement with a recent estimate [16], genomescale flux analysis revealed that about half of the available reactions ( $45 \%$ of the known metabolic genes) were not required for growth on glucose (Figure 1). Hence, deletion of these genes would not affect the growth phenotype on this substrate, making inactive reactions the primary reason for the apparent dispensability of genes with metabolic function. It should be noted that this apparent gene dispensability is a 
somewhat artificial classification that does not contribute to genetic robustness because most of these genes encode metabolic functions that are only relevant under conditions different from the one tested. The most important mechanism of true genetic robustness in yeast glucose metabolism was duplicate genes (Figure 7), the majority of which encoded essential reactions with no alternative pathway. Alternative pathways, contributed about $25 \%$ to genetic robustness by carbon flux rerouting. This leaves redundancy as the major, and modularity the minor, cause [50] of metabolic network robustness to single-gene deletions during growth on glucose.

\section{Materials and methods Yeast strains}

All prototrophic $S$. cerevisiae deletion mutants were constructed in the haploid, CEN.PK113-7D (Mata MAL2-8c SUC2) background with the homolog flanking region approach [17] (Table 1). Briefly, genomic DNA was isolated from the corresponding amino-acid auxotrophic mutants [23]. The kanMX4 cassettes of each mutant were amplified by PCR with primers located about 500 bp upstream and downstream of the deleted genes. The PCR reaction mixture was directly used for transformation and integrants were selected on YPD plates with $300 \mu \mathrm{g} / \mathrm{ml}$ geneticin. Correct cassette insertion was confirmed by overlapping PCR using either primer KanB (5'-CTGCAGCGAGGAGCCGTAAT-3') or KanC (5'-TGATTTTGATGACGAGCGTAA-3') primers in combination with one gene-specific primer. The reference strain was CEN.PK 113-7D with a deletion of the switching endonuclease, which was shown to be phenotypically neutral in chemostat competition experiments [51] and is commonly used as reference [52,53].

\section{Media and growth conditions}

The composition of the yeast minimal medium (MM) was

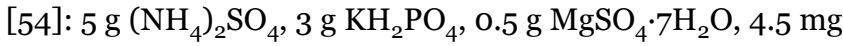
$\mathrm{ZnSO}_{4} \cdot 7 \mathrm{H}_{2} \mathrm{O}, 0.3 \mathrm{mg} \mathrm{CoCl} \cdot 6 \mathrm{H}_{2} \mathrm{O}$, $1.0 \mathrm{mg} \mathrm{MnCl}_{2} \cdot 4 \mathrm{H}_{2} \mathrm{O}, 0.3$ mg CuSO $4 \cdot 5 \mathrm{H}_{2} \mathrm{O}, 4.5 \mathrm{mg} \mathrm{CaCl} \cdot 2 \mathrm{H}_{2} \mathrm{O}$, 3.0 mg FeSO${ }_{4} \cdot 7 \mathrm{H}_{2} \mathrm{O}$, $0.4 \mathrm{mg} \mathrm{NaMoO} \cdot 2 \mathrm{H}_{2} \mathrm{O}$, $1.0 \mathrm{mg} \mathrm{H}_{3} \mathrm{BO}_{3}$, $0.1 \mathrm{mg} \mathrm{KI}, 15 \mathrm{mg}$ EDTA, $0.05 \mathrm{mg}$ biotin, $1.0 \mathrm{mg}$ calcium pantothenate, $1.0 \mathrm{mg}$ nicotinic acid, $25 \mathrm{mg}$ inositol, $1.0 \mathrm{mg}$ pyridoxine, $0.2 \mathrm{mg} p$ aminobenzoic acid, and $1.0 \mathrm{mg}$ thiamine. The medium was buffered at $\mathrm{pH} 5.0$ with $100 \mathrm{mM} \mathrm{KH}$-phthalate to reduce $\mathrm{pH}$ changes throughout the growth experiments to 0.05. Filtersterilized glucose and geneticin $(300 \mu \mathrm{g} / \mathrm{ml})$ were added freshly to the media. Batch growth experiments $(1.2 \mathrm{ml})$ were carried out in deep-well plates (System Duetz, Kühner AG, Switzerland) using an orbital shaker with $5 \mathrm{~cm}$ amplitude at $300 \mathrm{rpm}$ to allow optimal mixing [22].

Qualitative testing of mutant growth on glucose was done on agar plates. For this purpose, we used the haploid yeast mutant library in the BY4741 strain (MATa his3 41 leu2 $\Delta O$ met15 $\Delta$ o ura3 $\Delta o$ ) [23]. The composition of the yeast minimal medium for the plate growth assay was as described above
[54] with the exception of $20 \mathrm{~g} / \mathrm{l}$ agar for solidification. Glucose was added to a final concentration of $20 \mathrm{~g} / \mathrm{l}$. Strain auxotrophies were complemented with $20 \mathrm{mg} / \mathrm{l}$ histidine, uracil, methionine, lysine and $60 \mathrm{mg} / \mathrm{l}$ leucine. The plates were incubated at $30^{\circ} \mathrm{C}$ for 3 days before scoring of the growth phenotype and further incubated for 1 week to score slowgrowth phenotype mutants.

\section{Analytical procedures and ${ }^{13} \mathrm{C}$-labeling experiments} Cell growth was monitored by following optical density changes at a wavelength of $600 \mathrm{~nm}\left(\mathrm{OD}_{600}\right)$. Aliquots for extracellular metabolite analysis were centrifuged at 14,000 rpm in an Eppendorf tabletop centrifuge to remove cells. Glucose, acetate, ethanol and glycerol concentrations in the supernatant were determined with commercial enzymatic kits (Scil Diagnostics, Germany). Organic acids were quantified by high-pressure liquid chromatography (HPLC) using a Supelcogel C8 ( 4.6 by $250 \mathrm{~mm}$ ) ion-exclusion column. The column was eluted at $30^{\circ} \mathrm{C}$ with $2 \%$ sulfuric acid at a flow rate of $0.3 \mathrm{ml} / \mathrm{min}$. The organic acids were detected using a PerkinElmer UV detector (Series 2000) at a wavelength of 210 $\mathrm{nm}$. The physiological parameters maximum specific growth rate, biomass yield on glucose, and specific glucose consumption rate were calculated during the exponential growth phase.

All labeling experiments were carried out in batch cultures assuming pseudosteady-state conditions during the exponential growth phase $[12,55]$. ${ }^{13 C}$-labeling of proteinogenic amino acids was achieved either by growth on $5 \mathrm{~g} / \mathrm{l}$ glucose as a mixture of $80 \%(\mathrm{w} / \mathrm{w})$ unlabeled and $20 \%(\mathrm{w} / \mathrm{w})$ uniformly labelled [U-13C]glucose (13C > 99\%; Martek Biosciences, Columbia, MD) or 100\% [1-13C]glucose (> 99\%; Omicron Biochemicals, South Bend, IN). Cells from overnight cultures were harvested by centrifugation and washed using sugar-free MM to remove residual unlabeled carbon sources. Cultures were routinely inoculated to an maximum $\mathrm{OD}_{600}$ of 0.03 and harvested by centrifugation at an $\mathrm{OD}_{600} \leq$ 1. Residual medium was removed by washing the pellet with water. Cell protein was hydrolyzed for $24 \mathrm{~h}$ at $105^{\circ} \mathrm{C}$ in $6 \mathrm{M}$ HCL and dried in a heating block at $85^{\circ} \mathrm{C}$ for $6 \mathrm{~h}$. The free amino acids were derivatized at $85^{\circ} \mathrm{C}$ for $1 \mathrm{~h}$ using $15 \mu \mathrm{l}$ dimethylformamide and $15 \mu \mathrm{l} N$-(tert-butyldimethylsilyl)- $N$ methyl-trifluoroacetamide [10]. Gas chromatography-mass spectrometry (GC-MS) analysis was carried out as reported [12] using a series $8000 \mathrm{GC}$ in combination with an MD80o mass spectrometer (Fisons Instruments, Beverly, MA).

\section{Metabolic flux ratio analysis}

The recorded MS spectra include the distribution of mass isotopomers in 1-5 fragments of alanine, aspartate, glutamate, glycine, isoleucine, leucine, phenylalanine, proline, serine, threonine, tyrosine, and valine. For each amino-acid fragment $\alpha$, a mass isotopomer distribution vector (MDV) was assigned: 


$$
\operatorname{MDV}_{\alpha}=\left[\begin{array}{c}
\left(m_{o}\right) \\
\left(m_{1}\right) \\
\left(m_{2}\right) \\
\cdots \\
\left(m_{n}\right)
\end{array}\right] \text { with } \sum \mathrm{m}_{\mathrm{i}}=1
$$

with $m_{\mathrm{O}}$ being the fractional abundance of the lowest mass and $m_{i>0}$ the abundances of molecules with higher masses. The $\mathrm{MDV}_{\alpha}$ values were corrected for naturally occurring stable isotopes [12] to obtain the exclusive mass isotopomer distributions of the carbon skeletons. The corrected $\mathrm{MDV}_{\alpha}$ were used to calculate the amino acids $\left(\mathrm{MDV}_{\mathrm{AA}}\right)$ and metabolites $\left(\mathrm{MDV}_{\mathrm{M}}\right)$ mass distribution vectors. Ratios of converging intracellular fluxes to a given metabolite were calculated from the $\mathrm{MDV}_{\mathrm{M}}$ as described previously [12,29].

In addition, the relative contribution of the PP pathway was quantified from [1-13C]glucose experiments by tracking the positional $13 \mathrm{C}$-labeling $[10,56]$. The expected labeling pattern of triose phosphates or serine, which is derived exclusively through glycolysis, is $50 \%{ }^{13} \mathrm{C}$-label in the $\mathrm{C}_{1}$ positions. Hence, the fraction of serine derived through the pentose phosphate (PP) pathway can be derived according to Equation 2 [12]:

$$
\text { Serine through PP pathway }=1-\frac{\text { Serine13 }-\mathrm{GLU}_{3_{\text {unlabeled }}}}{0.5 \times\left(\mathrm{GLU}_{3} \text { unlabeled }_{\text {undabeled }} \times \mathrm{GLU}_{3_{1}}\right)-\mathrm{GLU}_{3_{\text {unlabe }}}}
$$

where $\mathrm{GLU}_{3} 3_{\text {unlabeled }}$ is an unlabeled three-carbon fragment from a source molecule of glucose. The remaining fraction of serine must then be derived through glycolysis. This flux ratio was not corrected for the potential withdrawal of $13 \mathrm{C}$-label in dihydroxyacetone-phosphate-based biomass synthesis (such as phospholipids) and glycerol formation [21], because the influence was negligible under the condition used. The largest effect was found in the mutant with the highest specific glycerol formation rate $(\operatorname{cox} 5 A)$, where the estimated relative flux through the PP pathway would decrease from $12 \pm 1 \%$ to $9 \pm$ $1 \%$.

\section{${ }^{13}$ C-constrained flux analysis}

Absolute values of intracellular fluxes were calculated with a flux model comprising all the major pathways of yeast central carbon metabolism (Additional data file 2). Deleted reactions were not omitted from the mutant models; thus the mutations were independently verified from the ${ }^{13 \mathrm{C}}$ data. The stoichiometric matrix of 34 linear equations and 30 metabolites has an infinite condition number [57]; it is thus underdetermined, and has a solution space with an infinite number of different flux vectors that fulfill the constraints from determined uptake and production rates. To uniquely solve the system for fluxes $(v)$, a set of linearly independent equations that quantify flux ratios (FlRs) were used to obtain eight constraints on the relative flux distribution from METAFoR analysis (see Additional data file 2).
The fraction of cytosolic oxaloacetate originating from cytosolic pyruvate is given by:

$$
F l R 1=\frac{v 23}{v 23+v 30}
$$

The fraction of mitochondrial oxaloacetate derived through anaplerosis is given by:

$$
F l R 2=\frac{v 29}{v 19+v 29}
$$

The fraction of PEP originating from cytosolic oxaloacetate is given by:

$F l R 3=\frac{v 22}{v 22+v 12}$

The fraction of serine derived through glycolysis is given by:

$$
F l R 4=\frac{2 v 4-v 6-v 7}{2 v 4+v 5+v 6}
$$

The upper and lower bounds for mitochondrial pyruvate derived through the malic enzyme (from mitochondrial malate) are given by:

$$
F l R_{5} \geq \frac{v 21}{v 32+v 21} \geq F l R 6
$$

The contribution of glycine to serine biosynthesis is given by:

$$
F l R 7=\frac{v 10}{v 10+v 8}
$$

and, finally, the contribution of serine to glycine biosynthesis is given by:

$$
F l R 8=\frac{v 9}{v 9+v 11}
$$

The stoichiometric matrix including Equations 3-10 has a condition number of 31 , implying that the model is numerically robust [57]. Error minimization was carried out as described by Fischer et al. [10]. Balanced NADPH production and consumption were not added as additional constraints. In general, NADPH production was constrained by Equations 3 and $7 / 8$, which estimate the relative use of the PP pathway and malic enzyme, respectively. As an additional source of $\mathrm{NADPH}$, the flux through the NADPH-dependent acetaldehyde dehydrogenase [33] was estimated from the acetate production rate and the biomass requirement for cytosolic acetyl-CoA. Deviation of the NADPH production estimated in this way from the consumption for biosynthesis was generally below $\pm 20 \%$, suggesting that the model assumptions and the experimental data are highly consistent. All extreme flux 
patterns were independently verified in 30-ml cultures (data not shown).

\section{Genome-scale flux analysis}

We used the experimentally determined in vivo flux data

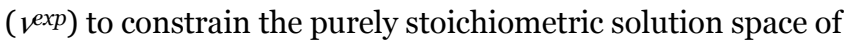
model iLL672 to obtain an experimentally validated genomescale wild-type flux solution $v^{W T}$. For glucose minimal medium, we constrained the model iLL672 with 30 fluxes that were derived from ${ }^{13 \mathrm{C}-l a b e l i n g}$ experiments [8]. In particular, we used ${ }^{13 C} \mathrm{C}$-constrained flux analysis [58] for GC-MSdetected mass isotope distributions in proteinogenic amino acids from a $20 \%$ [U-13C] glucose experiment and a compartmentalized yeast model [29]. These experimental data were to be kept within an accuracy $\delta$ of $\pm 10 \%$ when mapping the determined central metabolic fluxes to the genome-scale reference flux solution. To overcome mathematical artifacts such as futile cycling (that is, a closed loop of fluxes that bring no net change), the original linear programming problem was modified. A minimization of the Euclidean norm of fluxes was chosen as the objective function such that (s.t.) the mass balance equations hold:

$$
\begin{aligned}
& \min \sum_{i}\left|v_{i}^{W T}\right| \\
& \text { s.t. } \quad S \cdot v^{W T}=0 \\
& v_{l b, i}^{W T} \leq v_{i}^{W T} \leq v_{u b, i}^{W T} \\
& v_{j}^{\exp } \cdot(1-\delta) \leq v_{j}^{W T} \leq v_{j}^{\exp } \cdot(1+\delta)
\end{aligned}
$$

with $j$ as the set of experimentally determined fluxes. Reactions were categorized as flexible when fulfilling the following criteria: the reactions carried a non-zero flux; and the reaction was not essential for growth.

\section{In silico phenotyping of duplicate gene families}

Phenotype predictions of deletion mutants were analyzed computationally with FBA [3,59]. Assuming steady-state growth, mass balances were put up for each intracellular metabolite $M_{i}(1 \times n)$ that have to be fulfilled, when multiplied with the overall flux vector $v(n \times 1)$ :

$M_{i} \cdot v=0 . \quad(12)$

The entity of all $m$ metabolite mass balances yields the stoichiometric matrix $S(m \times n)$, where:

$\mathrm{S} \cdot v=0 . \quad(13)$

To pick one solution out of the overall solution space formed by the stoichiometric constraints, FBA generally assumes maximization of biomass growth $\mu$ as the global cellular goal [3,59]. Thus, the search for a single flux distribution $v$ results in a linear programming (LP) problem: $\max \mu$

$$
\begin{aligned}
& \text { s.t. } \quad \quad \quad S \cdot v=\mathrm{o} \\
& v_{l b, i} \leq v_{i} \leq v_{u b, \mathrm{i}},
\end{aligned}
$$

where $i=1, . ., M$ and $v_{l b, i}$ and $v_{u b, i}$ correspond to upper and lower bounds of a specific reaction $i$. Gene knockout mutants can be simulated easily in silico by setting the deleted reactions to zero. All LPproblems were solved using the opensource GNU linear programming kit [6o].

\section{Additional data files}

The following additional data are available with the online version of this paper. The classification of reactions according to Figure 1 is presented in Additional data file 1. The flux analysis model is defined in Additional data file 2. The physiological data, flux ratios and the calculated flux distributions are presented in Additional data file 3.

\section{Acknowledgements}

We are grateful to Eckhard Boles for providing the mael mutant. LarsM. Blank gratefully acknowledges financial support by the Deutsche Akademie der Naturforscher Leopoldina (BMBF-LPD/8-78).

\section{References}

I. Papin JA, Stelling J, Price ND, Klamt S, Schuster S, Palsson BO: Comparison of network-based pathway analysis methods. Trends Biotechnol 2004, 22:400-405.

2. Price ND, Reed JL, Palsson BO: Genome-scale models of microbial cells: evaluating the consequences of constraints. Nat Rev Microbiol 2004, 2:886-897.

3. Förster J, Famili I, Fu P, Palsson BO, Nielsen J: Genome-scale reconstruction of the Saccharomyces cerevisiae metabolic network. Genome Res 2003, I 3:244-253.

4. Reed JL, Palsson BO: Thirteen years of building constraintbased in silico models of Escherichia coli. J Bacteriol 2003, I 85:2692-2699.

5. Gu X: Evolution of duplicate genes versus genetic robustness against null mutations. Trends Genet 2003, I 9:354-356.

6. Hartman JLT, Garvik B, Hartwell L: Principles for the buffering of genetic variation. Science 200I, 29I:1001-1004.

7. Hellerstein MK: In vivo measurement of fluxes through metabolic pathways: the missing link in functional genomics and pharmaceutical research. Annu Rev Nutr 2003, 23:379-402.

8. Sauer U: High-throughput phenomics: experimental methods for mapping fluxomes. Curr Opin Biotechnol 2004, I 5:58-63.

9. Wiechert W: ${ }^{13}$ C metabolic flux analysis. Metab Eng 200I, 3:195-206.

10. Fischer E, Zamboni N, Sauer U: High-throughput metabolic flux analysis based on gas chromatography-mass spectrometry derived ${ }^{13} \mathrm{C}$ constraints. Anal Biochem 2004, 325:308-316.

II. Zamboni N, Sauer U: Model-independent fluxome profiling from ${ }^{2} \mathrm{H}$ and ${ }^{13} \mathrm{C}$ experiments for metabolic variant discrimination. Genome Biol 2004, 5:R99.

12. Fischer E, Sauer U: Metabolic flux profiling of Escherichia coli mutants in central carbon metabolism using GC-MS. Eur J Biochem 2003, 270:880-891.

13. Blank LM, Sauer U: TCA cycle activity in Saccharomyces cerevisiae is a function of the environmentally determined specific growth and glucose uptake rates. Microbiology 2004, I50:1085-1093.

14. Blank LM, Lehmbeck F, Sauer U: Metabolic flux and network analysis in fourteen hemiascomycetous yeasts. FEMS Yeast Res 2005, 5:545-558.

15. Duarte NC, Herrgard MJ, Palsson BO: Reconstruction and 
validation of Saccharomyces cerevisiae iND750, a fully compartmentalized genome-scale metabolic model. Genome Res 2004, I 4: I 298-1309.

16. Papp B, Pal C, Hurst LD: Metabolic network analysis of the causes and evolution of enzyme dispensability in yeast. Nature 2004, 429:661-664.

17. Wach A, Brachat A, Pohlmann R, Philippsen P: New heterologous modules for classical or PCR-based gene disruptions in Saccharomyces cerevisiae. Yeast 1994, 10:1793-1808.

18. van Dijken JP, Bauer J, Brambilla L, Duboc P, Francois JM, Gancedo C, Giuseppin ML, Heijnen JJ, Hoare M, Lange HC, et al.: An interlaboratory comparison of physiological and genetic properties of four Saccharomyces cerevisiae strains. Enzyme Microb Technol 2000, 26:706-7|4.

19. Pronk JT: Auxotrophic yeast strains in fundamental and applied research. Appl Environ Microbiol 2002, 68:2095-2 I 00.

20. Steinmetz LM, Scharfe C, Deutschbauer AM, Mokranjac D, Herman ZS, Jones T, Chu AM, Giaever G, Prokisch H, Oefner PJ, et al:: Systematic screen for human disease genes in yeast. Nat Genet 2002, 31 I:400-404.

21. Zamboni N, Fischer E, Laudert D, Aymerich S, Hohmann HP, Sauer $U$ : The Bacillus subtilis yqjl gene encodes the NADP+-dependent 6-P-gluconate dehydrogenase in the pentose phosphate pathway. J Bacteriol 2004, I 86:4528-4534.

22. Duetz WA, Ruedi L, Hermann R, O'Connor K, Buchs J, Witholt B: Methods for intense aeration, growth, storage, and replication of bacterial strains in microtiter plates. Appl Environ Microbiol 2000, 66:264I-2646.

23. Giaever G, Chu AM, Ni L, Connelly C, Riles L, Veronneau S, Dow S, Lucau-Danila A, Anderson K, Andre B, et al.: Functional profiling of the Saccharomyces cerevisiae genome. Nature 2002, 418:387-39l.

24. Winzeler EA, Liang H, Shoemaker DD, Davis RW: Functional analysis of the yeast genome by precise deletion and parallel phenotypic characterization. Novartis Found Symp 2000, 229: I05- 109. discussion I09-III.

25. Monschau N, Stahmann KP, Sahm H, McNeil JB, Bognar AL: Identification of Saccharomyces cerevisiae GLYI as a threonine aldolase: a key enzyme in glycine biosynthesis. FEMS Microbiol Lett 1997, 150:55-60.

26. Gombert AK, Moreira dos Santos M, Christensen B, Nielsen J: Network identification and flux quantification in the central metabolism of Saccharomyces cerevisiae under different conditions of glucose repression. J Bacteriol 200I, I 83: | 44|-|45I.

27. Maaheimo H, Fiaux J, Cakar ZP, Bailey JE, Sauer U, Szyperski T: Central carbon metabolism of Saccharomyces cerevisiae explored by biosynthetic fractional ${ }^{13} \mathrm{C}$ labeling of common amino acids. Eur J Biochem 200I, 268:2464-2479.

28. Szyperski T: Biosynthetically directed fractional ${ }^{13} \mathrm{C}$-labeling of proteinogenic amino acids. An efficient analytical tool to investigate intermediary metabolism. Eur J Biochem 1995, 232:433-448.

29. Blank LM, Sauer U: TCA cycle activity in Saccharomyces cerevisiae is a function of the environmentally determined specific growth and glucose uptake rates. Microbiology 2004, I50:1085-1093.

30. Sauer U, Hatzimanikatis V, Bailey JE, Hochuli M, Szyperski T, Wüthrich K: Metabolic fluxes in riboflavin-producing Bacillus subtilis. Nat Biotechnol 1997, 1 5:448-452.

31. Dos Santos MM, Gombert AK, Christensen B, Olsson L, Nielsen J: Identification of in vivo enzyme activities in the cometabolism of glucose and acetate by Saccharomyces cerevisiae by using (I3)C-labeled substrates. Eukaryotic Cell 2003, 2:599-608.

32. Meaden PG, Dickinson FM, Mifsud A, Tessier W, Westwater J, Bussey H, Midgley M: The ALD6 gene of Saccharomyces cerevisiae encodes a cytosolic, $\mathrm{Mg}(2+)$-activated acetaldehyde dehydrogenase. Yeast 1997, 13:1319-1327.

33. Grabowska D, Chelstowska A: The ALD6 gene product is indispensable for providing NADPH in yeast cells lacking glucose6-phosphate dehydrogenase activity. I Biol Chem 2003, 278: I3984-I3988.

34. Boubekeur S, Bunoust O, Camougrand N, Castroviejo M, Rigoulet M, Guerin B: A mitochondrial pyruvate dehydrogenase bypass in the yeast Saccharomyces cerevisiae. J Biol Chem 1999, 274:21044-21048.

35. Outten CE, Culotta VC: A novel NADH kinase is the mitochondrial source of NADPH in Saccharomyces cerevisiae. EMBO J 2003, 22:20I5-2024.
36. McEwen JE, Cumsky MG, Ko C, Power SD, Poyton RO: Mitochondrial membrane biogenesis: characterization and use of pet mutants to clone the nuclear gene coding for subunit $\mathbf{V}$ of yeast cytochrome c oxidase. J Cell Biochem 1984, 24:229-242.

37. McAlister-Henn L, Thompson LM: Isolation and expression of the gene encoding yeast mitochondrial malate dehydrogenase. J Bacteriol 1987, 169:5157-5166.

38. Schuster S, Fell DA, Dandekar T: A general definition of metabolic pathways useful for systematic organization and analysis of complex metabolic networks. Nat Biotechnol 2000, I 8:326-332.

39. Stelling J, Klamt S, Bettenbrock K, Schuster S, Gilles ED: Metabolic network structure determines key aspects of functionality and regulation. Nature 2002, 420:190-193.

40. Segre D, Vitkup D, Church GM: Analysis of optimality in natural and perturbed metabolic networks. Proc Natl Acad Sci USA 2002, 99:15112-15117.

4I. Covert MW, Knight EM, Reed JL, Herrgard MJ, Palsson BO: Integrating high-throughput and computational data elucidates bacterial networks. Nature 2004, 429:92-96.

42. Segre D: The regulatory software of cellular metabolism. Trends Biotechnol 2004, 22:261-265.

43. Dunn B, Ferea T, Spellman P, Schwarz J, Terraciano J, Troyanovich J, Walker S, Greene J, Shaw K, DiDomenico B, et al.: Genetic footprinting: a functional analysis of the $S$. cerevisiae genome. [http://www.yeastgenome.org].

44. Wu M, Tzagoloff A: Mitochondrial and cytoplasmic fumarases in Saccharomyces cerevisiae are encoded by a single nuclear gene FUMI. J Biol Chem I 987, 262: I 2275- 12282.

45. Van Maris AJ, Luttik MA, Winkler AA, Van Dijken JP, Pronk JT: Overproduction of threonine aldolase circumvents the biosynthetic role of pyruvate decarboxylase in glucose-limited chemostat cultures of Saccharomyces cerevisiae. Appl Environ Microbiol 2003, 69:2094-2099.

46. Winzeler EA, Shoemaker DD, Astromoff A, Liang $H$, Anderson K, Andre B, Bangham R, Benito R, Boeke JD, Bussey H, et al.: Functional characterization of the $S$. cerevisiae genome by gene deletion and parallel analysis. Science 1999, 285:901-906.

47. Nowak MA, Boerlijst MC, Cooke J, Smith JM: Evolution of genetic redundancy. Nature 1997, 388: I67-I71.

48. Wagner A: Robustness against mutations in genetic networks of yeast. Nat Genet 2000, 24:355-36I.

49. Gu Z, Steinmetz LM, Gu X, Scharfe C, Davis RW, Li WH: Role of duplicate genes in genetic robustness against null mutations. Nature 2003, 42 I:63-66.

50. Stelling J, Sauer U, Szallasi Z, Doyle FJ 3rd, Doyle J: Robustness of cellular functions. Cell 2004, I I 8:675-685.

5I. Baganz F, Hayes A, Marren D, Gardner DC, Oliver SG: Suitability of replacement markers for functional analysis studies in Saccharomyces cerevisiae. Yeast 1997, 13:1563-1573.

52. Allen J, Davey HM, Broadhurst D, Heald JK, Rowland JJ, Oliver SG, Kell DB: High-throughput classification of yeast mutants for functional genomics using metabolic footprinting. Nat Biotechnol 2003, 2 I:692-696.

53. Raamsdonk LM, Teusink B, Broadhurst D, Zhang N, Hayes A, Walsh MC, Berden JA, Brindle KM, Kell DB, Rowland JJ, et al.: A functional genomics strategy that uses metabolome data to reveal the phenotype of silent mutations. Nat Biotechnol 200I, 1 9:45-50.

54. Verduyn C, Postma E, Scheffers WA, Van Dijken JP: Effect of benzoic acid on metabolic fluxes in yeasts: a continuous-culture study on the regulation of respiration and alcoholic fermentation. Yeast 1992, 8:50|-5I7.

55. Sauer U, Lasko DR, Fiaux J, Hochuli M, Glaser R, Szyperski T, Wüthrich K, Bailey JE: Metabolic flux ratio analysis of genetic and environmental modulations of Escherichia coli central carbon metabolism. J Bacteriol 1999, 181:6679-6688.

56. Christensen B, Christiansen T, Gombert AK, Thykaer J, Nielsen J: Simple and robust method for estimation of the split between the oxidative pentose phosphate pathway and the Embden-Meyerhof-Parnas pathway in microorganisms. Biotechnol Bioeng 200I, 74:517-523.

57. Nissen TL, Schulze U, Nielsen J, Villadsen J: Flux distributions in anaerobic, glucose-limited continuous cultures of Saccharomyces cerevisiae. Microbiology 1997, I43:203-218.

58. Fischer E, Zamboni N, Sauer U: High-throughput metabolic flux analysis based on gas chromatography-mass spectrometry derived ${ }^{13} \mathrm{C}$ constraints. Anal Biochem 2004, 325:308-316.

59. Edwards JS, Palsson BO: The Escherichia coli MGI655 in silico 
metabolic genotype: its definition, characteristics, and capabilities. Proc Natl Acad Sci USA 2000, 97:5528-5533.

60. Makhorin A: GNU Linear Programming Kit 200I [http://www.gnu.org/ software/glpk/glpk.html]. Moscow, Russia: Moscow Aviation Institute

6I. Yeast Deletion Project and Proteomics of Mitochondria

Database [http://www-deletion.stanford.edu/YDPM] 